\title{
Magnetospheric energy inputs into the upper atmospheres of the giant planets
}

\author{
C. G. A. Smith, S. Miller, and A. D. Aylward \\ Atmospheric Physics Laboratory, Department of Physics and Astronomy, University College, London, UK
}

Received: 10 February 2005 - Revised: 13 April 2005 - Accepted: 14 April 2005 - Published: 28 July 2005

\begin{abstract}
We revisit the effects of Joule heating upon the upper atmospheres of Jupiter and Saturn. We show that in addition to direct Joule heating there is an additional input of kinetic energy - ion drag energy - which we quantify relative to the Joule heating. We also show that fluctuations about the mean electric field, as observed in the Earth's ionosphere, may significantly increase the Joule heating itself. For physically plausible parameters these effects may increase previous estimates of the upper atmospheric energy input at Saturn from $\sim 10 \mathrm{TW}$ to $\sim 20 \mathrm{TW}$.
\end{abstract}

Keywords. Ionosphere (Electric fields and currents; Planetary ionosphere) - Magnetospheric physics (Auroral phenomena)

\section{Introduction}

All four of the giant planets exhibit high thermospheric temperatures, the origin of which has yet to be thoroughly explained. Measurements by the Voyager missions (reviewed by Atreya, 1986) and the Galileo probe (Seiff et al., 1997) indicate temperatures of $\sim 900 \mathrm{~K}$ or greater throughout Jupiter's thermosphere. Modelling indicates that such high temperatures cannot be explained by solar heating alone (Strobel and Smith, 1973). Similar discrepancies are observed at Saturn, Uranus and Neptune (Yelle and Miller, 2004).

Attempts to understand these high temperatures have generally focussed on the heating effects of breaking gravity and acoustic waves (Young et al., 1997; Matcheva and Strobel, 1999; Hickey et al., 2000; Schubert et al., 2003), on energy deposited by auroral particle precipitation (Grodent et al., 2001), on Joule heating (Cowley et al., 2004) and on the transfer of energy from large-scale neutral winds, driven by ion drag (Miller et al., 2000; Millward et al., 2005). In this letter we present two new perspectives on this problem.

Correspondence to: C. G. A. Smith

(chriss@apl.ucl.ac.uk)
Firstly, we quantify the input of bulk kinetic energy associated with Joule heating (which we term "ion drag energy"), and show that it may be an important energy source; secondly, we show that fluctuations in the electric field may significantly increase the Joule heating itself.

\section{General situation}

Plasma flows in the magnetosphere or at the magnetosphere/solar wind boundary generate electric fields that map along magnetic field lines into the ionosphere. The nature of these motions have been sketched for both Jupiter (Cowley et al., 2003) and Saturn (Cowley et al., 2004). We refer the reader to these papers for a detailed discussion. For our purposes it is sufficient to note that to a good approximation the plasma simply subcorotates, to a greater or lesser extent, with respect to the planet. From a thermospheric perspective this results in an approximately westwards "ion wind", such as has been observed on both Jupiter (Stallard et al., 2001) and Saturn (Stallard et al., 2004).

In the upper atmosphere, the relative motions of the neutral and ionised gases has two effects. Firstly, some of the kinetic energy of both components is thermalised, heating the atmosphere. This is the "Joule heating" (Cowley et al., 2004). Secondly, there is an exchange of momentum, and thus an exchange of kinetic energy, between the two components. If, in the appropriate frame of reference, the ions move with a greater velocity than the neutrals, the neutrals will gain kinetic energy (Miller et al., 2000; Millward et al., 2005). This is often the case at high latitudes if one considers the frame of reference corotating with the planet. It is this energy input that we seek to quantify. We refer to it as "ion drag energy".

Note that the Joule heating is a frame-invariant quantity because it represents changes in the random thermal motions of the gas. These must be identical in all frames. However, the ion drag energy is frame-variant because the bulk kinetic energy of the gas is itself a frame-variant quantity. 


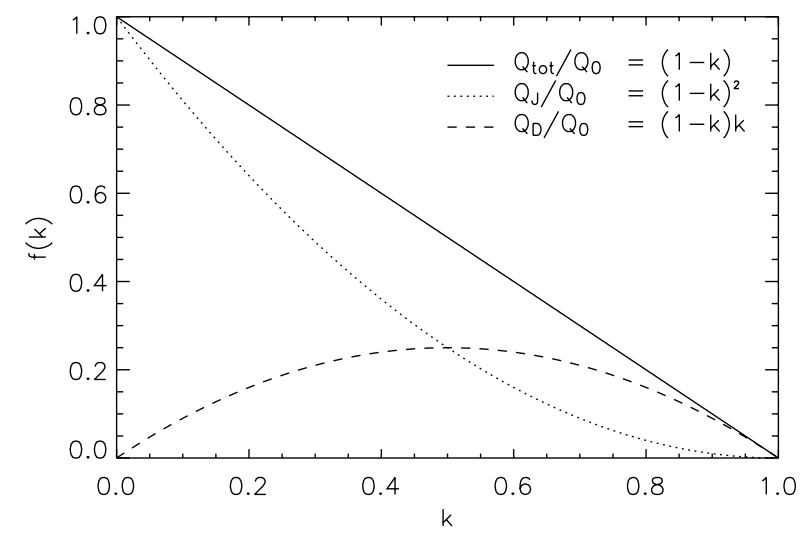

Fig. 1. Dependence of $Q_{t o t} / Q_{0}, Q_{J} / Q_{0}$ and $Q_{D} / Q_{0}$ upon $k$.

\section{Ion drag}

We now support the qualitative arguments presented above with some simple theoretical considerations. Before considering the specific cases of Jupiter and Saturn, we state general expressions for the Joule heating $Q_{J}$ and the ion drag energy $Q_{D}$ in a general frame $S$. The total work done on the upper atmosphere by the magnetospheric electric fields is:

$Q_{\text {tot }}=\boldsymbol{j} \cdot \boldsymbol{E}$

where $\boldsymbol{j}$ is the current and $\boldsymbol{E}$ is the electric field. In the general frame $S, Q_{t o t}=Q_{J}+Q_{D}$.

The $\boldsymbol{j} \times \boldsymbol{B}$ force exerted by the magnetospheric electric fields accelerates the neutral gas. The associated rate of kinetic energy input, which we call the "ion drag energy" $Q_{D}$ is then:

$Q_{D}=\boldsymbol{v}_{\boldsymbol{n}} \cdot \boldsymbol{j} \times \boldsymbol{B}$

where $\boldsymbol{v}_{\boldsymbol{n}}$ is the neutral wind velocity and $\boldsymbol{B}$ is the magnetic field. The Joule heating $Q_{J}$ is then simply $Q_{t o t}-Q_{D}$ :

$$
\begin{aligned}
Q_{J} & =\boldsymbol{j} \cdot \boldsymbol{E}-\boldsymbol{v}_{\boldsymbol{n}} \cdot \boldsymbol{j} \times \boldsymbol{B} \\
& =\boldsymbol{j} \cdot \boldsymbol{E}+\boldsymbol{j} \cdot \boldsymbol{v}_{\boldsymbol{n}} \times \boldsymbol{B} \\
& =\boldsymbol{j} \cdot\left(\boldsymbol{E}+\boldsymbol{v}_{\boldsymbol{n}} \times \boldsymbol{B}\right) \\
\Rightarrow \quad Q_{J} & =\boldsymbol{j} \cdot \boldsymbol{E}^{\prime}
\end{aligned}
$$

where we have introduced the electric field transformed into the frame of the neutral wind $\boldsymbol{E}^{\prime}=\boldsymbol{E}+\boldsymbol{v}_{\boldsymbol{n}} \times \boldsymbol{B}$. This quantity is uniquely defined because it represents the electric field in the special frame $S^{\prime}$ in which the neutrals are at rest. Thus $Q_{J}=\boldsymbol{j} \cdot \boldsymbol{E}^{\prime}$ is frame-invariant. In this frame, $S^{\prime}$, we have by definition $\boldsymbol{v}_{\boldsymbol{n}}=0$ and thus $Q_{D}$ is zero.

We now apply the above equations to an idealised model of the northern auroral regions of the giant planets. We assume an upwards vertical component of the magnetic field, $+B$, and an equatorwards-directed electric field, $+E$, in the frame of reference that corotates with the planet. This configuration will generate a westwards ion wind whose magnitude is $v_{i}=E / B$. The westwards velocity of the neutral gas is $v_{n}$.
Thus $B, E$ and $v_{n}$ are mutually perpendicular. The electric field drives an equatorwards current:

$j=\sigma_{P} E^{\prime}$

where $\sigma_{P}$ is the local Pedersen conductivity and $E^{\prime}=E-v_{n} B$ is the electric field in the rest frame $S^{\prime}$ of the neutrals. We can write:

$E^{\prime}=E-v_{n} B=E\left(1-\frac{v_{n}}{E / B}\right)=E(1-k)$

where we have followed Millward et al. (2005) in defining k:

$k=\frac{v_{n}}{E / B}=\frac{v_{n}}{v_{i}}$

Thus, from Eqs. 4, 1, 3 and 2 respectively:

$j=(1-k) \sigma_{P} E$

$Q_{t o t}=j E=(1-k) \sigma_{P} E^{2}$

$Q_{J}=j E^{\prime}=(1-k)^{2} \sigma_{P} E^{2}$

$Q_{D}=v_{n} j B=k(1-k) \sigma_{P} E^{2}$

For simplicity, we now define $Q_{0}=\sigma_{P} E^{2}$. Figure 1 shows how the three quantities $Q_{J} / Q_{0}, Q_{D} / Q_{0}$ and $Q_{t o t} / Q_{0}$ vary with $k$. It is clear that if the Joule heating $Q_{J}$ is considered alone, it is strongly dependent upon $k$ through the factor $(1-k)^{2}$. If $k$ is as great as 0.5 then $Q_{J}$ falls to only $25 \%$ of $Q_{0}$, its value when the neutral wind is zero.

However, the addition of the ion drag energy means that $Q_{t o t}$ varies only as $(1-k)$ (Eq. 8). The result of this is a much less rapid decrease in energy input, such that if $k$ is as great as 0.7 , as predicted in some circumstances by the JIM model (Millward et al., 2005), the energy input including ion drag is $\sim 3$ times that expected from Joule heating alone.

Recent modelling of the Jovian upper atmosphere (Millward et al., 2005) has demonstrated that neutral winds driven by ions accelerated in a constant electric field reach a steady velocity after approximately $30 \mathrm{~min}$. After this time, although the ions are continuously imparting kinetic energy to the neutrals, the latter do not continue to accelerate. The conclusion is that the ion drag must be continuously viscously dissipated, heating the thermosphere in the process. Similar processes are expected to operate in the case of Saturn and, perhaps, other giant planets.

\section{Electric field variability}

The best available quantitative models of the plasma flows in the polar regions of Jupiter and Saturn (e.g. Nichols and Cowley, 2004; Cowley et al., 2004) represent fields that are both axisymmetric and time-averaged. However, it has been shown for Earth that electric fields may fluctuate on timescales of less than a minute (Codrescu et al., 2000; Aruliah et al., 2005). The magnitude of these fluctuations may be comparable to, or greater than, the magnitude of the electric 
field itself. As we show below, the effect of these fluctuations is to increase the time-averaged Joule heating, as first pointed out for Earth by Codrescu et al. (1995).

Suppose that the electric field $E$ described above represents the mean of a fluctuating electric field

$$
E_{f}(t)=E(1+f(t))
$$

where the fluctuations $f(t)$ are distributed randomly with a mean $\bar{f}=0$ and variance $\overline{f^{2}}=s^{2}$. Since it has been shown (Millward et al., 2005) that the neutral velocity $v_{n}$ responds relatively slowly to an applied electric field, we can consider it to be a constant. The fluctuating rest-frame field $E_{f}^{\prime}(t)$ is:

$$
\begin{aligned}
E_{f}^{\prime}(t) & =E_{f}(t)-v_{n} B=E(1+f(t)-k) \\
& =E[(1-k)+f(t)]
\end{aligned}
$$

We now modify Eqs. 7, 9 and 10 to take account of the fluctuations, indicating fluctuating variables with the subscript $f$ :

$j_{f}(t)=[(1-k)+f(t)] \sigma_{P} E$

$Q_{J f}(t)=j_{f}(t) E_{f}^{\prime}(t)=[(1-k)+f(t)]^{2} Q_{0}$

$Q_{D f}(t)=v_{n} j_{f}(t) B=k[(1-k)+f(t)] Q_{0}$

Expanding and time averaging Eqs. 14 and 15 yields:

$$
\begin{aligned}
\overline{Q_{J f}} & =\left[(1-k)^{2}+2(1-k) \bar{f}+\overline{f^{2}}\right] Q_{0} \\
& =\left[(1-k)^{2}+s^{2}\right] Q_{0} \\
\overline{Q_{D f}} & =k[(1-k)+\bar{f}] Q_{0} \\
& =k(1-k) Q_{0}
\end{aligned}
$$

It is clear that $\overline{Q_{D f}}=Q_{D}$, i.e. the ion drag energy is entirely unaffected by the fluctuating electric field. However, $\overline{Q_{J f}}=Q_{J}+s^{2} Q_{0}$, such that the Joule heating itself may be significantly increased depending on the value of $s$. For brevity we write this "fluctuation energy" as $Q_{F}=s^{2} Q_{0}$. It is important to point out that the contribution due to the fluctuations does not depend upon the factor $k$. If, as discussed above, $k$ is as great as 0.7 , fluctuations with $s \sim 1$ would represent the dominant component of the Joule heating. It is also worth noting that the above derivation does not depend on the timescale of the electric field fluctuations other than that it must be short enough that the neutral atmosphere is unable to respond.

In Fig. 2 we illustrate the relative importance of the energy inputs $Q_{J}, Q_{D}$ and $Q_{F}$ in the parameter space of $s$ and $k$. It is clear that $Q_{J}$ is the most important energy source if $s$ and $k$ are both small. Ion drag is only important if $k$ is large and $s$ is small. In general different points in the atmosphere will correspond to different points in this parameter space, because $k$ varies with position. Huang and Hill (1989) found $k$ to increase monotonically with height; in this case fluctuations and ion drag would become more important with increasing altitude. In contrast, Millward et al. (2005) found that $k$ peaked in the low-altitude conducting layer of the ionosphere, producing the opposite situation; further work is required to understand these differences.

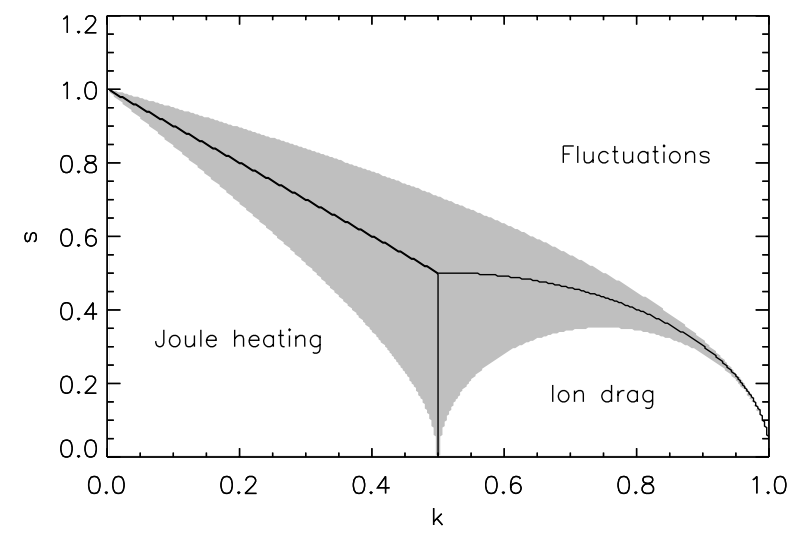

Fig. 2. Relative importance of energy sources as a function of $k$ and $s$. The marked areas represent regions of parameter space in which each energy source is the most important. In the unshaded regions the marked energy source accounts for greater than $50 \%$ of the total energy input.

\section{Saturn}

In the specific case of Saturn, recent work by Cowley et al. (2004) used empirical models of the magnetosphere to derive the expected magnetospheric energy inputs. They calculated a height-integrated quantity $p_{J}^{*}$, which they referred to as the "effective Joule heating". Using their notation:

$p_{J}^{*}=(1-k) \Sigma_{P} E^{2}$

where $\Sigma_{P}$ is the height-integrated Pedersen conductivity. They found a globally integrated $p_{J}^{*}$ of about $10 \mathrm{TW}$, concentrated poleward of $25^{\circ}$ colatitude in each hemisphere. They state that the height-integrated Joule heating $p_{J}$ is given by:

$p_{J}=(1-k)^{2} \Sigma_{P} E^{2}=(1-k) p_{J}^{*}$

Thus they can only estimate the Joule heating to within the unknown factor $(1-k)$. However, we can now add the heightintegrated ion drag energy $p_{T}$ :

$p_{T}=k(1-k) \Sigma_{P} E^{2}=k p_{J}^{*}$

such that the total height-integrated energy input $p_{t o t}$ (neglecting fluctuations) is:

$p_{\text {tot }}=[(1-k)+k] p_{J}^{*}=p_{J}^{*}$

Thus the total energy input (neglecting fluctuations) is given by the empirically derived quantity $p_{J}^{*}$, which turns out to be the height-integrated analogue of our $Q_{t o t}$.

We may now include the component from fluctuating electric fields, $p_{F}=s^{2} p_{J}$. From Eqs. 19 and 20 it is then easily shown that the total time-averaged energy input is:

$\overline{p_{\text {totf }}}=p_{J}^{*}\left[1+\frac{s^{2}}{1-k}\right]$

The factor in square brackets represents a correction to the energy input $p_{J}^{*}$ calculated from the time-averaged field. 
Taking nominal values of $k=0.5$ (Millward et al., 2005) and $s=1$ (by analogy with Earth; Codrescu et al., 2000), the energy input increases from $\sim 10 \mathrm{TW}$ to $\sim 20 \mathrm{TW}$, made up of $\sim 5 \mathrm{TW}$ of mean-field Joule heating, $\sim 5 \mathrm{TW}$ of ion drag, and $\sim 10 \mathrm{TW}$ from the fluctuation term. It can be seen that in this case the original mean-field estimate of Joule heating is only $25 \%$ of the total energy input.

\section{Discussion and conclusions}

We have shown that in addition to direct Joule heating the ionospheric current systems at Jupiter and Saturn supply a comparable quantity of kinetic energy to the upper atmosphere through ion drag. We have also shown that fluctuations about the time-averaged electric field have the potential to increase the total Joule heating considerably. To understand the relative effects of these energy sources we require further knowledge of the parameters $k$ and $s$. We expect, due to the complex nature of the Jovian and Kronian magnetospheres, that both parameters will be strongly dependent upon magnetic latitude.

Past determinations of $k$ have been based purely on modelling, since no measurements of high-latitude thermospheric winds exist for either Jupiter or Saturn. There seems little hope of this situation improving in the foreseeable future.

For $s$, we have taken a nominal value of $s=1$ by analogy with the terrestrial results of Codrescu et al. (2000). Their study focused on the polar cap region in which the plasma flows are dominated by the Dungey cycle. For Jupiter and Saturn it seems reasonable to expect similar behaviour in the Dungey cycle regions. However, the plasma flows equatorwards of these regions are dominated by subcorotation of the magnetosphere associated with mass loading from moons and rings. It may not be appropriate to extrapolate the terrestrial results to these environments. Saur et al. (2002) have shown that the middle magnetosphere of Jupiter does exhibit turbulence, although the extent to which this turbulence maps to a fluctuating electric field in the ionosphere is a question which is beyond the scope of this letter.

We also note that if significant turbulence were to exist in the inner magnetosphere or plasmasphere regions this could result in a fluctuating electric field at mid-latitudes where the mean electric field is very small. This could lead to a very large increase in Joule heating at mid-latitudes. If present, this effect could offer a potentially simple explanation of the observed temperatures, since it would not be necessary to invoke redistribution of energy from the polar regions.

\section{References}

Aruliah, A. L., Griffin, E. M., Aylward, A. D., Ford, E. A. K., Kosch, M. J., Davis, C. J., Howells, V. S. C., Pryse, S. E., Middleton, H. R., and Jussila, J.: First direct evidence of meso-scale variability on ion-neutral dynamics using co-located tristatic FPIs and EISCAT radar in Northern Scandinavia, Ann. Geo- phys., 23, 147-162, 2005,

\section{SRef-ID: 1432-0576/ag/2005-23-147.}

Atreya, S. K.: Atmospheres and Ionospheres of the Outer Planets and their Satellites, Springer-Verlag, 1986.

Codrescu, M. V., Fuller-Rowell, T. J., and Foster, J. C.: On the importance of E-field variability for Joule heating in the highlatitude thermosphere, Geophys. Res. Lett., 22, 2393-2396, 1995.

Codrescu, M. V., Fuller-Rowell, T. J., Foster, J. C., Holt, J. M., and Cariglia, S. J.: Electric field variability associated with the Millstone Hill electric field model, J. Geophys. Res., 5265-5274, 2000.

Cowley, S. W. H., Bunce, E. J., Stallard, T. S., and Miller, S.: Jupiter's polar ionospheric flows: theoretical interpretation, Geophys. Res. Lett., 30(5), 1220, doi:10.1029/2002GL016030, 2003.

Cowley, S. W. H., Bunce, E. J., and O'Rourke, J. M.: A simple quantitative model of plasma flows and currents in Saturn's polar ionosphere, J. Geophys. Res., 109(A05212), doi:10.1029/2003JA010375, 2004.

Grodent, D., Waite, J. H., and Gérard, J.: A self-consistent model of the Jovian auroral thermal structure, J. Geophys. Res., 12 93312 952, 2001.

Hickey, M. P., Walterscheid, R. L., and Schubert, G.: Gravity Wave Heating and Cooling in Jupiter's Thermosphere, Icarus, 148, 266-281, 2000.

Huang, T. S. and Hill, T. W.: Corotation lag of the Jovian atmosphere, ionosphere, and magnetosphere, J. Geophys. Res., 94, 3761-3765, 1989.

Matcheva, K. I. and Strobel, D. F.: Heating of Jupiter's Thermosphere by Dissipation of Gravity Waves Due to Molecular Viscosity and Heat Conduction, Icarus, 140, 328-340, 1999.

Miller, S., Achilleos, N., Ballester, G. E., Geballe, T. R., Joseph, R. D., Prange, R., Rego, D., Stallard, T., Tennyson, J., Trafton, L. M., and Waite, J. H.: The role of $\mathrm{H}_{3}^{+}$in planetary atmospheres, Royal Society of London Philosophical Transactions Series A, 358, 2485-2502, 2000.

Millward, G., Miller, S., Stallard, T., Achilleos, N., and Aylward, A. D.: On the dynamics of the jovian ionosphere and thermosphere IV: Ion-neutral Coupling, Icarus, 173, 200-211, 2005.

Nichols, J. and Cowley, S.: Magnetosphere-ionosphere coupling currents in Jupiter's middle magnetosphere: effect of precipitation-induced enhancement of the ionospheric Pedersen conductivity, Ann. Geophys., 22, 1799-1827, 2004,

SRef-ID: 1432-0576/ag/2004-22-1799.

Saur, J., Politano, H., Pouquet, A., and Matthaeus, W. H.: Evidence for weak MHD turbulence in the middle magnetosphere of Jupiter, Astronomy and Astrophysics, 386, 699-708, 2002.

Schubert, G., Hickey, M. P., and Walterscheid, R. L.: Heating of Jupiter's thermosphere by the dissipation of upward propagating acoustic waves, Icarus, 163, 398-413, 2003.

Seiff, A., Kirk, D. B., Knight, T. C. D., Young, L. A., Milos, F. S., Venkatapathy, E., Mihalov, J. D., Blanchard, R. C., Young, R. E., and Schubert, G.: Thermal structure of Jupiter's upper atmosphere derived from the Galileo probe, Science, 276, 102-104, 1997.

Stallard, T., Miller, S., Millward, G., and Joseph, R. D.: On the Dynamics of the Jovian Ionosphere and Thermosphere. I. The Measurement of Ion Winds, Icarus, 154, 475-491, 2001.

Stallard, T. S., Miller, S., Trafton, L. M., Geballe, T. R., and Joseph, R. D.: Ion winds in Saturn's southern auroral/polar region, Icarus, 167, 204-211, 2004. 
Strobel, D. F. and Smith, G. R.: On the Temperature of the Jovian Thermosphere, Journal of Atmospheric Science, 30, 718-725, 1973.

Yelle, R. V. and Miller, S.: The Thermosphere and Ionosphere of Jupiter, in Jupiter: Planet, Satellites and Magnetosphere, (Eds.) Bagenal, F., McKinnon, W., and Dowling, T. Cambridge University Press, 185-218, 2004.
Young, L. A., Yelle, R. V., Young, R., Seiff, A., and Kirk, D. B.: Gravity Waves in Jupiter's Thermosphere, Science, 276, 108111, 1997. 